\title{
ORIGINAL RESEARCH \\ Long-term morbidity and mortality in survivors of critical illness: a 5-year observational follow-up study
}

\author{
PJ Secombe, P Stewart
}

Alice Springs Hospital, Alice Springs, Northern Territory, Australia

Submitted: 5 February 2016; Revised: 26 September 2016; Accepted: 18 October 2016; Published: 23 February 2017

\section{Secombe PJ, Stewart $P$}

Long-term morbidity and mortality in survivors of critical illness: a 5-year observational follow-up study Rural and Remote Health 17: 3908. (Online) 2017

Available: http://www.rrh.org.au

\section{A B S T R A C T}

Introduction: This prospective observational study over 5 years aimed to quantify long-term morbidity and mortality in a prospectively recruited cohort of Central Australian survivors of critical illness.

Methods: Eligible participants are survivors of an intensive care unit (ICU) admission for a critical illness at the Alice Springs Hospital (ASH), prospectively recruited during 2009. The ASH ICU is a 10-bed unit located in Central Australia with approximately 600 admissions annually, 95\% of which are emergent, and 65\% Indigenous. All-cause mortality, secondary healthcare utilisation and functional outcomes were measured by 6-minute walk distance (an indicator of functional status) and the home and community care (HACC) screening tool at 5 years.

Results: Sixty eight percent of the cohort had died at 5 years. Median age of death was 53 years with a median time to death of 604 days following ICU admission. There was increased secondary healthcare utilisation measured by emergency department presentations and hospital re-admissions, with a median 5.22 healthcare presentations per year alive. There is evidence of ongoing functional limitation with 6-minute walk distance at 5 years significantly less than that predicted, despite high scores on the HACC screening assessment suggesting virtually full resumption of basic and domestic activities of daily living.

Conclusions: A critical illness is not an isolated event, and there is evidence of ongoing high secondary healthcare utilisation, reflecting a high burden of disease. Mortality in this cohort is higher than would be expected from international data, and at a young median age, suggesting significant loss of productive life years. In addition, there is evidence of ongoing morbidity, with higher rates of healthcare utilisation than comparable international studies. This has profound implications for healthcare planners due to the ongoing economic implications, and may suggest a need for increased primary healthcare resources to pre-emptively manage chronic disease and reduce the burden of healthcare utilisation at acute care facilities. 
Key words: activities of daily living, Australia, critical care, critical illness, follow-up studies, intensive care units, outcome assessment (health care), patient readmission, survivors, longitudinal studies, survival rate.

\section{Introduction}

This article reports on the 5-year outcomes of a cohort prospectively recruited as part of exploratory research examining functional outcomes following a life-threatening illness ${ }^{1}$. Alice Springs Hospital (ASH) Intensive Care Unit (ICU) services a unique population. The catchment area exceeds 1 million $\mathrm{km}^{2}$, and contains an estimated population of 40000 , approximately $40 \%$ of whom identify as Indigenous ${ }^{2}$. This produces a unique case mix characterised by a high proportion of Indigenous patients requiring emergent admission (Table 1), and unique mortality outcomes (Fig1) ${ }^{3}$.

Despite over a decade of political rhetoric and increased funding there continues to be a substantial 'gap' between life expectancy for Indigenous and non-Indigenous Australians ${ }^{1}$. In addition to the excess mortality, there is substantial morbidity, with increased rates of hospitalisation for preventable disease ${ }^{4}$. Although there is some evidence the gap may be closing, many Closing the Gap targets remain behind schedule, including that of lowering death rates, while funding in real terms has fallen ${ }^{5-7}$.

ICU mortality, particularly for sepsis-related admissions, has decreased over the past decade, but there continues to be insufficient information about the outcomes of survivors ${ }^{4,5}$. What little data exists has been derived from large tertiary institutions in capital cities, and demonstrate persisting functional deficits, and increased rates of healthcare utilisation $^{8-12}$. There is little on the outcomes for regional and rural patients.

The authors have previously reported on the poor outcomes at 6 months in a cohort prospectively recruited following survival of a life-threatening illness ${ }^{1,13}$. This research showed excess mortality, reduced 6-minute walk distances, and evidence of a persisting inflammatory response, despite return to a functional state as assessed by basic measures of activity of daily living ${ }^{1,13}$. The 5 -year outcomes of the same cohort are presented in this article.

\section{Methods}

This prospective longitudinal observational study was conducted in the ASH ICU, a regional medical and surgical ICU that has approximately 600-700 admissions annually, of whom approximately $25 \%$ require mechanical ventilation for a mean period of approximately 4.5 days ${ }^{14}$.

\section{Participants}

Patients identified as high risk were prospectively recruited to the original study when eligibility criteria were met (Box $1)^{1,13}$. Recruitment to the study occurred between February and August 2009, with follow-up occurring at 6 months (previously reported) and at 5 years ${ }^{1,13}$.

Eligibility for the current 5-year follow-up included the same criteria. Patients who had previously declined consent, or who were missed in the original study, were re-contacted and invited to participate.

\section{Outcomes}

Primary outcome was all-cause mortality at 5 years, ascertained by review of hospital records and crossreferenced with the Northern Territory Births, Deaths and Marriages Register. Secondary outcomes focused on secondary healthcare utilisation (emergency department (ED) presentations and hospital admissions), as well as function (6minute walk distance and activities of daily living). 
Table 1: Patient data for Alice Springs Hospital Intensive Care Unit versus comparable rural/regional hospitals ${ }^{3,14}$

\begin{tabular}{|c|c|c|}
\hline Data $^{\dagger}$ & ASH ICU & ANZICS data \\
\hline Age (years) & $48(35-60)$ & $63(45-5)^{4}$ \\
\hline Length of stay (days) & $2.1(1.0-3.8)$ & $1.8(0.9-3.3)^{9}$ \\
\hline APACHE III & $40(27-56)$ & $43(27-60)^{n}$ \\
\hline SMR & $0.36(0.18-0.64)$ & $0.86(0.82-0.91)^{\natural}$ \\
\hline Emergency admission (\%) & 95 & $45^{\S}$ \\
\hline Indigenous (\%) & 65 & $5.6^{\S}$ \\
\hline \multicolumn{3}{|c|}{$\begin{array}{l}\text { Data is presented as median (and interquartile range), or absolute number (and/or percentage) } \\
\text { Comparable regional ANZICS ICUs } \\
\text { \$ All ANZICS ICUs }\end{array}$} \\
\hline
\end{tabular}

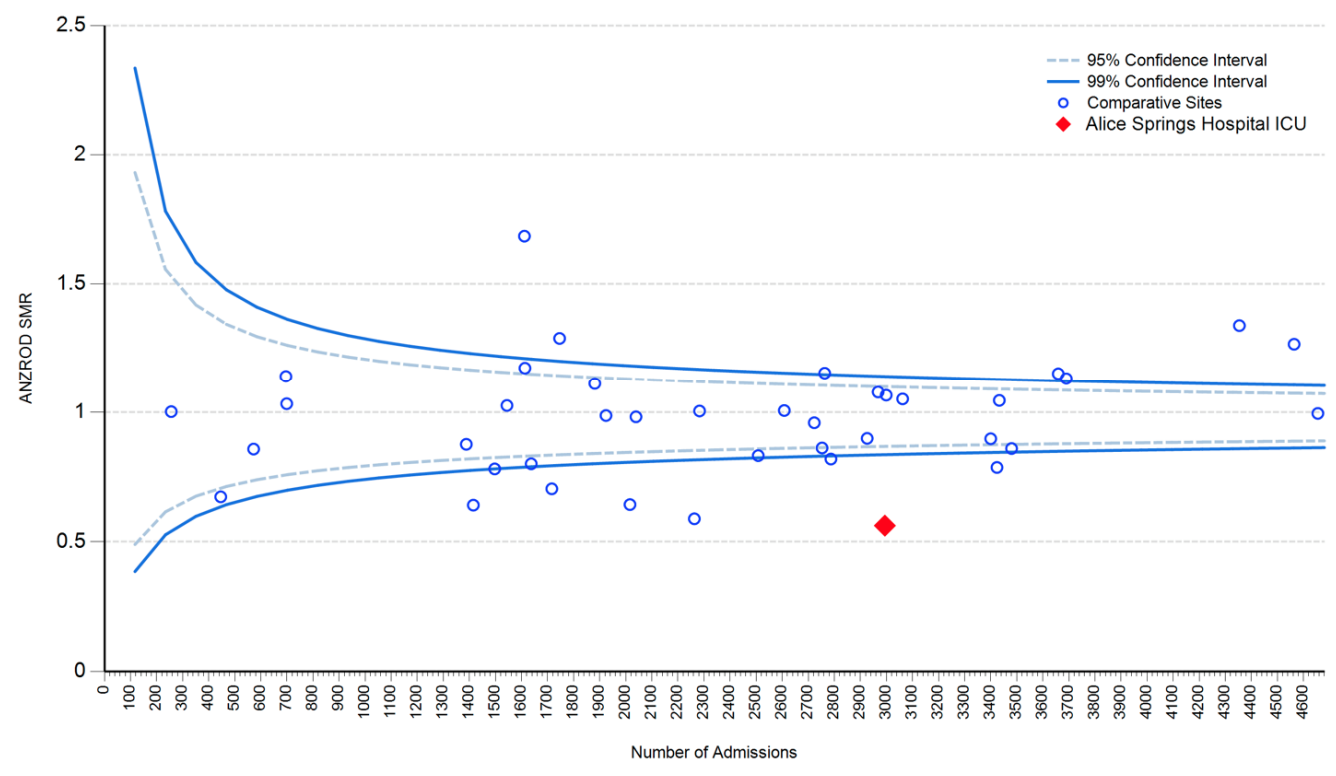

ANZROD SMR, Australian New Zealand Risk of Death Standardised Mortality Ratio. ICU, intensive care unit.

Figure 1: Standardised mortality ratio funnel plot for Alice Springs Hospital Intensive Care Unit and comparative sites, 2009-2014 ${ }^{3}$.

\section{Tools for assessment}

A composite measure of secondary healthcare utilisation was derived by combining ED presentations and hospital readmissions. Admissions to the ED short stay unit for overnight admission were coded as an ED presentation, while
ED presentations that resulted in hospital admission were coded as a hospital admission only. Re-admissions and ED presentations for Northern Territory residents were ascertained by cross-referencing with the Northern Territory Government Hospital Information System, while participants who had relocated interstate were asked to catalogue their 
presentations to hospital via questionnaire. Given that mortality could skew the results of healthcare utilisation (since a deceased patient cannot interact with the healthcare system), the number of presentations was divided by the survival time to produce the number of healthcare presentations per year alive. Subsequent separate analyses of ED presentations and hospital admission were undertaken since these have substantially different economic implications, and are a crude measure of the degree of physiological disturbance.

The 6-minute walk distance has been well studied and is recognised as an easy, cheap, well-tolerated assessment that provides an indication of functional status, as well as predicting morbidity and mortality for cardio-respiratory disease. Reference equations exist that predict performance ${ }^{15-}$

17. The 6-minute walk distance was calculated at hospital discharge, 6 months and at 5 years.

The home and community care (HACC) functional assessment scale is used as a screening tool to indicate the need for further functional assessment ${ }^{18}$. It comprises a questionnaire that identifies deficiencies in functional status either basic activities of daily living, including washing oneself and dressing, or domestic activities of daily living, including managing finances, medications and shopping.

\section{Statistical methods}

Median results are reported due to the small sample numbers. Variables were analysed using appropriate nonparametric tests including the Mann-Whitney U-test for ordinal data, the Wilcoxon matched pairs test for repeated measurements, and Yates corrected $\chi^{2}$ for nominal data using Statistica (Statsoft Incorporated, http://www.statsoft.com). A result was considered significant if $p<0.05$.

\section{Ethics approval}

The study received ethics approval from the Central Australian Human Research Ethics Committee (HREC-13157). Informed consent was obtained from patients.

\section{Results}

There were 25 eligible patients identified, including 18 patients who had previously consented and were involved in the original research (Fig2) ${ }^{1,13}$. Tables 2 and 3 provide the demographic background, including the Charlson CoMorbidity Index (CCMI), which is a method of classifying and quantifying co-morbid disease in longitudinal studies and also allows comparison with similar cohorts, and ICU course $^{19,20}$. As per ethics requirement (Human Research Ethics Committee 13-157), in order to avoid distress to family and community, the health record was first reviewed, the local clinic contacted, and cross-referencing with the Northern Territory Births, Deaths and Marriages Register was undertaken for all participants before contact was attempted. This allowed identification of those who had died during the follow-up period. Two patients who were homeless and living in the Alice Springs area were contacted by the ASH Aboriginal Liaison Service, but could not be followed up. These patients were deemed to have been lost to follow-up, but were known to be alive at the follow-up census date. Similarly, three (all non-Indigenous) patients had relocated interstate. All of these patients were personally contacted by the primary author, two consented to involvement and returned questionnaires sent to them. The third verbally consented to involvement, but failed to return her consent form and was deemed to have been lost to follow-up.

\section{Mortality}

Fifteen deaths were identified from case record review and cross-referencing with the Northern Territory Births, Deaths and Marriages Register, 7 (47\%) of whom had died within 6 months of ICU discharge (Table 4). Median age at death was 53 years (interquartile range (IQR) 39-71 years), and median time to death was 604 days (IQR 55-1691 days). Figure 3 shows the survival curve for the cohort and Table 5 the outcomes of individual patients. The sample size was considered too small to conduct subgroup analysis. 


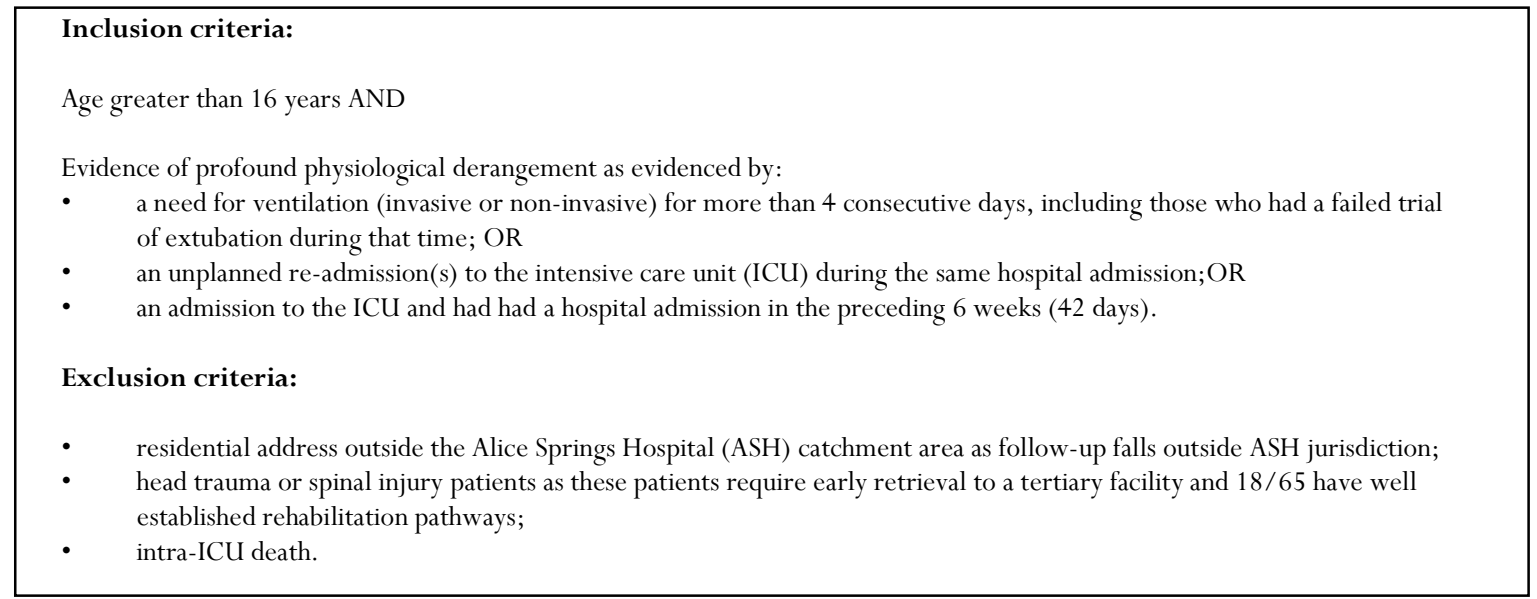

\section{Box 1: Study inclusion and exclusion criteria}

Table 2: Demographic characteristics of eligible patients

\begin{tabular}{|l|c|}
\hline Demographic characteristic $^{\dagger}$ & \multicolumn{1}{|c|}{ Frequency $^{\prime}$} \\
\hline Indigenous & $19(86)$ \\
\hline Male & $65(43)$ \\
\hline Age at follow-up (years) & $6(27)$ \\
\hline Homeless & $2(1-3)$ \\
\hline CCMI & $11(50)$ \\
\hline Diabetic (type II) & $8(36)$ \\
\hline History of alcohol use & $4(18)$ \\
\hline Tobacco smoker & $19(41)$ \\
\hline Chronic kidney disease & \\
\hline
\end{tabular}

${ }^{\dagger}$ Data is presented as median (and IQR), or absolute number (and percentage).

CCMI, Charlson Co-Morbidity Index. IQR, interquartile range.

Table 3: Alice Springs Hospital Intensive Care Unit admission details and admission course of eligible patients

\begin{tabular}{|l|c|}
\hline Admission detail & Frequency \\
\hline Emergency admission & $22(100)$ \\
\hline After hours admission & $16(73)$ \\
\hline Medical admission & $17(77)$ \\
\hline Surgical admission & $5(23)$ \\
\hline Sepsis contributing to admission & $17(77)$ \\
\hline APACHE II & $19(15-25)$ \\
\hline Ventilated (hours) & $140(64-410)$ \\
\hline Acute kidney injury & $12(55)$ \\
\hline ICU length of stay (days) & $6(5-16)$ \\
\hline Hospital length of stay (days) & $7(6-25)$ \\
\hline Time to 5-year review (days) & $1822(1759-1886)$ \\
\hline
\end{tabular}

${ }^{\dagger}$ Data is presented as median (and IQR), or absolute number (and percentage).

APACHE, Acute Physiology and Chronic Health Evaluation. ICU, intensive care unit. IQR, interquartile range. 


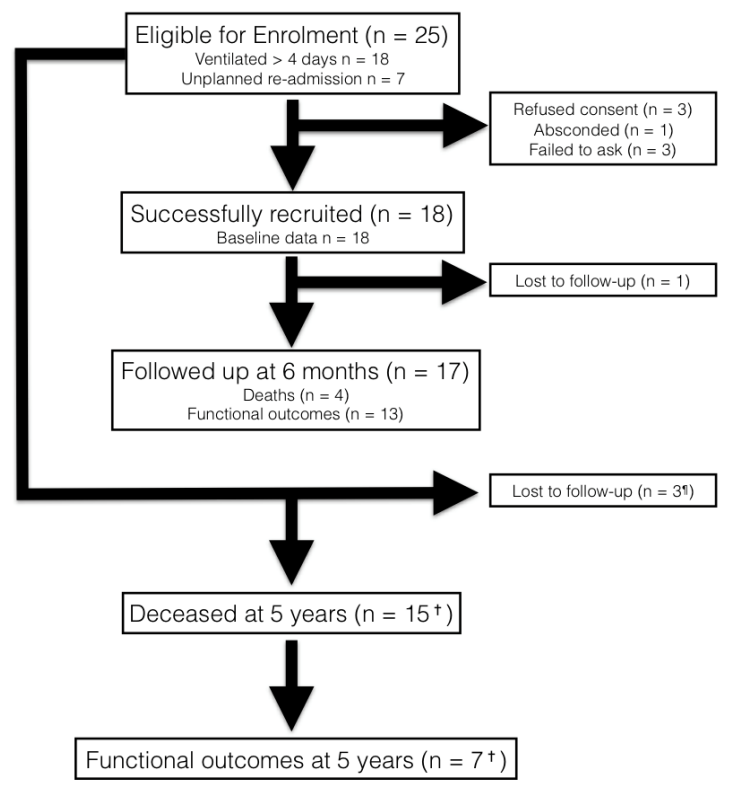

${ }^{\dagger}$ All patients who were eligible for the original study were re-contacted.

" Contact made with all three patients. Two Indigenous patients were living Alice Springs, but were homeless and declined. One non-Indigenous patient was contacted and verbally consented, but failed to return consent form.

Figure 2: Flow diagram for eligible patients.Table 4: Outcomes of eligible patients

\begin{tabular}{|l|c|}
\hline Outcome $^{\dagger}$ & Frequency \\
\hline Deceased & $15(68)$ \\
\hline Age at death (years) & $53(39-71)$ \\
\hline Survival time (days) $(n=15)$ & $604(55-1691)$ \\
\hline Achieved 6MWD (metres) $(n=5)$ & $124(120-306)$ \\
\hline Predicted 6MWD (metres) $(n=5)$ & $517(479-534)$ \\
\hline 6MWD difference (metres) $(n=5)$ & $407(297-410)$ \\
\hline HACC BADL score at 5 years $(n=8)$ & $20(19.5-20)$ \\
\hline HACC DADL score at 5 years $(n=8)$ & $27.5(19.5-29)$ \\
\hline Total healthcare presentations $(n=22)$ & $6.0(3-21)$ \\
\hline Healthcare presentations/year alive $(n=22)$ & $5.22(1.0-10.7)$ \\
\hline Re-admissions $(n=22)$ & $5(1-13)$ \\
\hline Time to first re-admission $($ days $)(n=18)$ & $279(21-1498)$ \\
\hline Re-admissions/year alive $(n=22)$ & $2.35(0.4-6.3)$ \\
\hline ED presentations $(n=22)$ & $2(0-6)$ \\
\hline Time to first ED presentation $($ days $)(n=16)$ & $71(27.5-735)$ \\
\hline ED presentations/year alive $(n=22)$ & $0.71(0.0-3.2)$ \\
\hline
\end{tabular}

${ }^{\dagger}$ Data is presented as median (and IQR), or absolute number (and percentage).

6MWD, 6-minute walk distance. BADL, basic activities of daily living. DADL, domestic activities of daily living. ED, emergency department. HACC, home and community care. IQR, interquartile range. 
Figure 3: Survival curve for eligible patients.

\begin{tabular}{|c|c|c|c|c|c|c|c|}
\hline Patient & $\begin{array}{c}\text { ICU admission } \\
\text { date }\end{array}$ & Admission diagnosis & CCMI & $\begin{array}{l}\text { Consented } \\
\text { in } 2009\end{array}$ & Outcome & $\begin{array}{l}\text { Consented } \\
\text { in } 2014\end{array}$ & Outcome \\
\hline LW & $15 / 1 / 09$ & Trauma & 0 & $\mathrm{Y}$ & Alive & & Lost to follow-up ${ }^{\dagger}$ \\
\hline AnD & $24 / 1 / 09$ & Trauma & 0 & $\mathrm{Y}$ & Alive & & Deceased \\
\hline LD & $11 / 2 / 09$ & Sepsis & 1 & $\mathrm{~N}$ & & & Deceased \\
\hline $\mathrm{SP}$ & $17 / 2 / 09$ & Bronchiectasis & 2 & $\mathrm{Y}$ & Deceased & & Deceased \\
\hline JW & $1 / 3 / 09$ & Altered conscious state & 2 & $\mathrm{Y}$ & Alive & & Deceased \\
\hline SR & $9 / 3 / 09$ & Gastrointestinal bleed & 4 & $\mathrm{Y}$ & Alive & & Deceased \\
\hline $\mathrm{DF}$ & $17 / 3 / 09$ & Bronchiectasis & 3 & $\mathrm{Y}$ & Alive & & Deceased \\
\hline GR & $18 / 3 / 09$ & Sepsis & 0 & $\mathrm{Y}$ & Alive & & Lost to follow-up ${ }^{\dagger}$ \\
\hline TR & $2 / 4 / 09$ & Sepsis & 4 & FTA & & & Deceased \\
\hline $\mathrm{MJ}$ & $12 / 4 / 09$ & CCF & 3 & $\mathrm{Y}$ & Alive & $\mathrm{Y}$ & Alive \\
\hline TT & $12 / 4 / 09$ & $\mathrm{CCF}$ & 3 & $\mathrm{Y}$ & Alive & & Deceased \\
\hline AlD & $10 / 5 / 09$ & Sepsis & 1 & $\bar{Y}$ & Lost to follow-up & $\bar{Y}$ & Alive \\
\hline NJ & $13 / 5 / 09$ & Bronchiectasis & 4 & $\mathrm{Y}$ & Deceased & & Deceased \\
\hline DT & $6 / 6 / 09$ & Trauma & 0 & $\mathrm{~N}$ & & & Lost to follow-up ${ }^{\dagger}$ \\
\hline DJ & $26 / 6 / 09$ & Sepsis & 1 & FTA & & & Deceased \\
\hline RW & $27 / 6 / 09$ & COPD & 1 & $\mathrm{Y}$ & Alive & & Deceased \\
\hline NB & $27 / 6 / 09$ & COPD & 1 & $\mathrm{Y}$ & Deceased & & Deceased \\
\hline EL & $2 / 7 / 09$ & $\mathrm{CCF}$ & 3 & FTA & & & Deceased \\
\hline VP & $24 / 7 / 09$ & Pancreatitis & 2 & $\mathrm{~N}$ & & $\mathrm{Y}$ & Alive \\
\hline $\mathrm{RD}$ & $26 / 7 / 09$ & Cardiac arrest & 4 & $\mathrm{Y}$ & Alive & $\mathrm{Y}$ & Alive \\
\hline $\mathrm{ChR}$ & $29 / 7 / 09$ & Sepsis & 1 & ToL & & $\mathrm{Y}$ & Alive \\
\hline CoR & $1 / 8 / 09$ & Sepsis & 3 & $\mathrm{Y}$ & Alive & & Deceased \\
\hline $\mathrm{CS}$ & $7 / 8 / 09$ & Sepsis & 0 & $\mathrm{Y}$ & Alive & $\mathrm{Y}$ & Alive \\
\hline NS & $7 / 8 / 09$ & Sepsis & 4 & $\mathrm{Y}$ & Deceased & & Deceased \\
\hline $\mathrm{TC}$ & $11 / 8 / 09$ & Sepsis & 1 & $\mathrm{Y}$ & Alive & $\mathrm{Y}$ & Alive \\
\hline
\end{tabular}

${ }^{\dagger}$ Lost to follow-up, but known to be alive. LW personally contacted by lead author; GR and DT contacted by Alice Springs Hospital Aboriginal Liaison Service.

CCF, congestive cardiac failure. CCMI, Charlson Co-Morbidity Index. COPD, infective exacerbation chronic obstructive pulmonary disease. FTA, failed to seek consent. ICU, intensive care unit. ToL, took own leave (absconded).

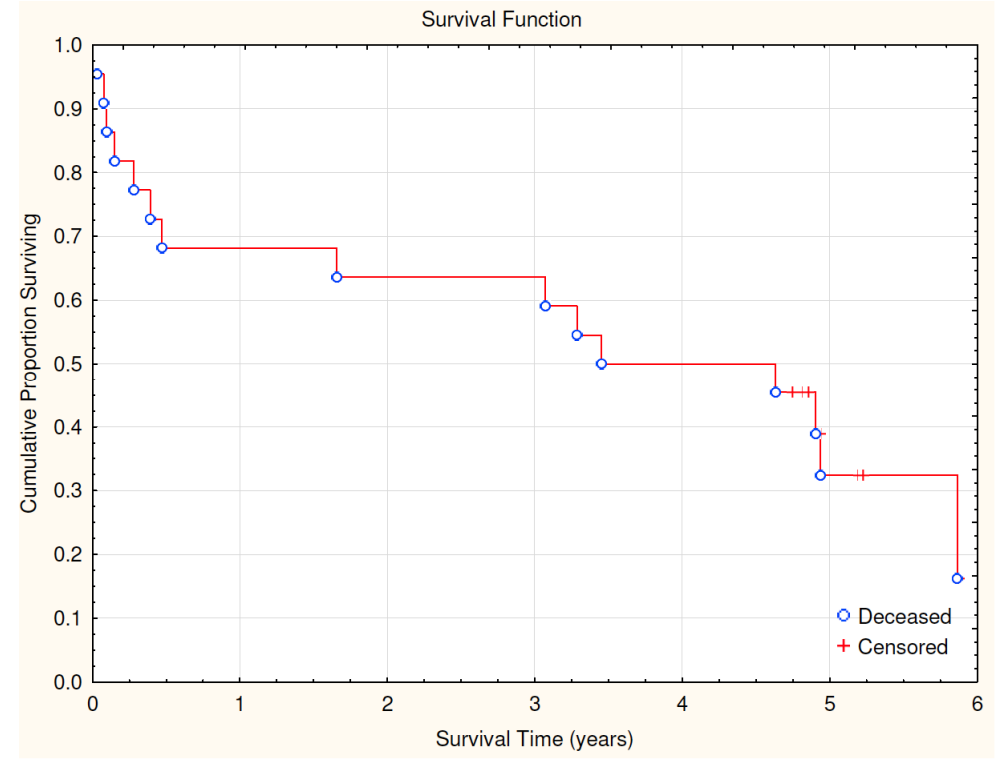

Figure 3: Survival curve for eligible patients. 


\section{Healthcare utilisation}

By the conclusion of the follow-up period, there was no patient who had not either died, or had an episode of secondary healthcare contact, resulting in 5.22 (IQR 1.0 10.7) healthcare presentations per year alive. Forty one percent of the cohort had more than 10 episodes of healthcare contact over the 5 -year follow up period (Fig4). The majority (76\%) of the cohort still alive had their first episode of healthcare utilisation in the first 12 months (Fig5).

When healthcare utilisation is broken down into the individual components, $82 \%$ of the cohort $(100 \%$ of those still alive) had been re-admitted at least once during the follow-up period with a median time to first re-admission of 279 days (IQR 21-1498 days). This group required a median five re-admissions (IQR 1-13) over the follow-up period, with 2.35 (0.4-6.3) re-admissions per year alive. Similarly, $73 \%$ of the cohort ( $96 \%$ of those still alive) had presented to an ED at least once during the follow-up period without requiring a hospital admission, with a median time to the first ED presentation of 71 days (IQR 27.5-735 days). This group had a median two ED presentations (IQR 0-6) during the 5year follow-up period, with 0.71 (IQR 0.0-3.2) ED presentations per year alive.

Table 6 describes the demographic, ICU course and outcome data for survivors and non-survivors. Neither the number of interactions with the healthcare system, the number of readmissions, nor the number of ED presentations was statistically different between surddvivors and non-survivors (Mann-Whitney U-test, $z=0.42, \quad p=0.67, \quad$ Mann-Whitney U-test, $z=0.07$, $p=0.94$; Mann-Whitney U-Test, $z=-0.46, \quad p=0.63$, respectively). Of interest, however, was that survivors at 5 years had a longer ICU length of stay than non-survivors (Table 6), albeit that this difference was not statistically significant (MannWhitney U-test, $z=-1.45, p=0.14)$.

\section{Functional outcomes}

Data for 6-minute walk distances were available for $71 \%$ of the patients remaining alive at 5 years. Two patients (29\%) were unable to undertake the task (one patient was blind, and one could not understand the task due to an acquired brain injury). These two patients were excluded from further analysis of the 6-minute walk distance. Of the remaining patients the median predicted 6-minute walk distance was $517 \mathrm{~m}$ (IQR 479-534 m), while the median 6-minute walk distance achieved was significantly shorter, at $124 \mathrm{~m}$ (IQR 120-306 m; Wilcoxon matched pairs $z=2.02, p<0.05)^{15-17}$. Figure 6 depicts median predicted and achieved 6 -minute walk distances over time.

The HACC screening tool demonstrated the cohort was able to manage basic activities of daily living soon after their hospital discharge, with a median score at 6 months of 20 (IQR 18-20), which remained unchanged to 5 years (median score 20 (IQR 19.5-20); Wilcoxon matched pairs, $z=0.80, p>0.05$ ). Similarly, domestic activities of daily living did not change significantly over the 5-year follow up period (median score 23.8 (IQR 23-30)) at 6 months compared to 24.8 (IQR 19.5-29) at 5 years; Wilcoxon matched pairs $z=0.00, p>0.05)$.

\section{Discussion}

This is the first article to the authors' knowledge that prospectively follows a heterogeneous cohort of survivors of critical illness to 5 years, and certainly the first from a regional setting. Amongst this cohort there is a high mortality rate amongst survivors of a critical illness (68\%), with high healthcare utilisation (5.22 healthcare presentations per year alive). There appears to be ongoing functional limitation despite high scores achieved with a screening tool that assesses activities of daily living, although the small numbers make conclusions about this difficult to draw.

These outcomes compare unfavourably with other published follow-up studies, including a recently published large retrospective comparative observational database review from Canada ${ }^{12}$. Hill's group followed up patients admitted to any ICU in Ontario between 2002 and 2012, examining longterm mortality and healthcare utilisation. Despite their cohort being older at admission there was a similar degree of concurrent illness as measured by the $\mathrm{CCMI}^{12}$. Post-hospital 
mortality in this group was reported to be $32 \%$, less than half that reported in the present study. Furthermore, healthcare utilisation as measured by ED presentations and hospital readmissions was lower in the cohort by Hill and colleagues, at $84.1 \%$ and $65.3 \%$ during the follow-up period.

Herridge and colleagues reported a 5-year mortality in their prospectively recruited cohort of acute respiratory distress syndrome survivors of $19.2 \%$, with a further $9 \%$ lost to follow-up ${ }^{9}$. Nesseler and colleagues, in the only other prospective study, report a 6 -month mortality of $34 \%$ in a cohort who survived an admission to single French ICU with septic shock ${ }^{21}$.

A number of retrospective database reviews inform outcomes in survivors. Prescott and colleagues interrogated the Health and Retirement Study database, a longitudinal panel study ${ }^{11}$. They report a 1 -year mortality of $44 \%$. Although this sample is older (mean age 78.6 years) than the cohort reported in this study, the CCMI was similar (mean score 2.1). Liu and colleagues, in a retrospective database analysis of patients admitted with sepsis to 21 community-based hospitals, report a 1 -year mortality of $9.5 \%{ }^{10}$. In both these studies a minority of patients were admitted to an ICU (38\% and 35\%, respectively).

Hicks and Mackle retrospectively examined the cause of death in patients surviving to hospital discharge in a New Zealand cohort ${ }^{22}$. This single centre retrospective study from a mixed ICU showed 1- and 2-year survival rates of $93.8 \%$ and 90.3\%, respectively ${ }^{12}$. Retrospective studies from separate ICUs in Western Australia also suggest a high survival rate. Hill and colleagues report a 12-month mortality of $12 \%$ in survivors of an ICU admission, and Williams and colleagues report a 5 -year mortality of $16.3 \%{ }^{23,24}$.

These suggest that the mortality rate in this cohort is higher than expected. The reason(s) underlying this high mortality rate are not clear, but factors possibly involved include the high numbers of Indigenous patients, compounded by a rural/remote setting, a high burden of chronic disease (as reflected by the high CCMI) despite a relatively young age, as well as poor access to and utilisation of primary healthcare services ${ }^{25-27}$.
Sepsis is already recognised as a leading contributor to productive years lost in the USA, and the median age of death in this cohort, in which sepsis contributed to the admission reason in $72 \%$ of cases, suggests that many are dying while still potentially highly productive members of their family and society ${ }^{28}$.

Hill and colleagues also found $82 \%$ of their Western Australian cohort required re-admission in the first 12 months after their index ICU admission ${ }^{23}$. The readmission rate reported by Hill and colleagues is consistent with international trends found in other retrospective studies. Prescott and colleagues found $60 \%$ of their cohort admitted to US hospitals required re-admission within 12 months, while Liu and colleagues found $48 \%$ of their sample required re-admission within 12 months ${ }^{10,11}$. Goodwin and colleagues reported $48 \%$ of their cohort who had survived an ICU admission for severe sepsis required re-admission by 6 months $^{29}$. Patients in all of these retrospective studies were older than this cohort, yet the re-admission rate and healthcare utilisation in this cohort from Central Australia is similar and confirm that the trends observed in the first twelve months continue out to 5 years. It also confirms that an ICU admission is not an isolated medical event, and that a considerable healthcare cost follows from surviving a critical illness. Hill and colleagues suggested that the 'time frame over which hospitalisations cluster either side of an ICU admission may extend to greater than 12 months', a contention that would be supported by this data ${ }^{23}$.

Although Herridge and colleagues described ongoing healthcare utilisation in their cohort of acute respiratory distress syndrome survivors, they report on healthcare costs, and not the number of interactions making comparison difficult ${ }^{9,30}$. They do report on 6-minute walk distances achieved at each of the follow-up time points. At 12 months they found survivors of ARDS achieved 66\% of predicted 6minute walk distance, which increased slightly at 5 years to $76 \%$ predicted $^{9,30}$. Although the number of participants who could complete the 6-minute walk distance was small in this cohort, they achieved only $30 \%$ and $24 \%$ of the predicted distance at 6 months and 5 years respectively, suggesting ongoing functional limitations, despite achieving high scores on a screening test for activities of daily living ${ }^{1}$. 
Table 6: Demographics and intensive care unit characteristics: survivors vs non-survivors

\begin{tabular}{|c|c|c|c|}
\hline Characteristic $^{\dagger}$ & $\begin{array}{c}\text { Non-survivors } \\
(n=15)\end{array}$ & $\begin{array}{c}\text { Survivors } \\
(n=7)\end{array}$ & $P$ \\
\hline Age at ICU admission (years) & $47(37-63)$ & $47(34-60)$ & 0.55 \\
\hline Male & $12(80)$ & $4(57)$ & 0.54 \\
\hline Indigenous & $14(93)$ & $5(71)$ & 0.47 \\
\hline Homeless & $4(26)$ & $2(29)$ & 0.61 \\
\hline Diabetic (type II) & $7(47)$ & $4(57)$ & 1.0 \\
\hline History of alcohol use & $4(27)$ & $4(57)$ & 0.36 \\
\hline Tobacco smoker & $3(20)$ & $1(14)$ & 0.79 \\
\hline ICU length of stay (days) & $5(4-16)$ & $10(6-22)$ & 0.14 \\
\hline APACHE II & $20(15-25)$ & $19(13-26)$ & 0.86 \\
\hline CCMI & $3(1-4)$ & $1(1-3)$ & 0.31 \\
\hline Medical admission & $13(87)$ & $4(57)$ & 0.09 \\
\hline Sepsis contributing to admission & 11 & 6 & 0.92 \\
\hline Healthcare presentations/year alive & $6.2(1.0-17.9)$ & $3.1(1.0-4.6)$ & 0.67 \\
\hline Re-admissions/year alive & $3.4(0-10.7)$ & $2.1(0.8-5.9)$ & 0.64 \\
\hline ED presentations/year alive & $0.6(0.0-2.0)$ & $1.2(0.2-4.6)$ & 0.94 \\
\hline
\end{tabular}

${ }^{\dagger}$ Data is presented as median (and IQR), or absolute number (and percentage).

APACHE, Acute Physiology and Chronic Health Evaluation. CCMI, Charlson Co-Morbidity Index. ED, emergency department. ICU, intensive care unit. IQR, interquartile range.

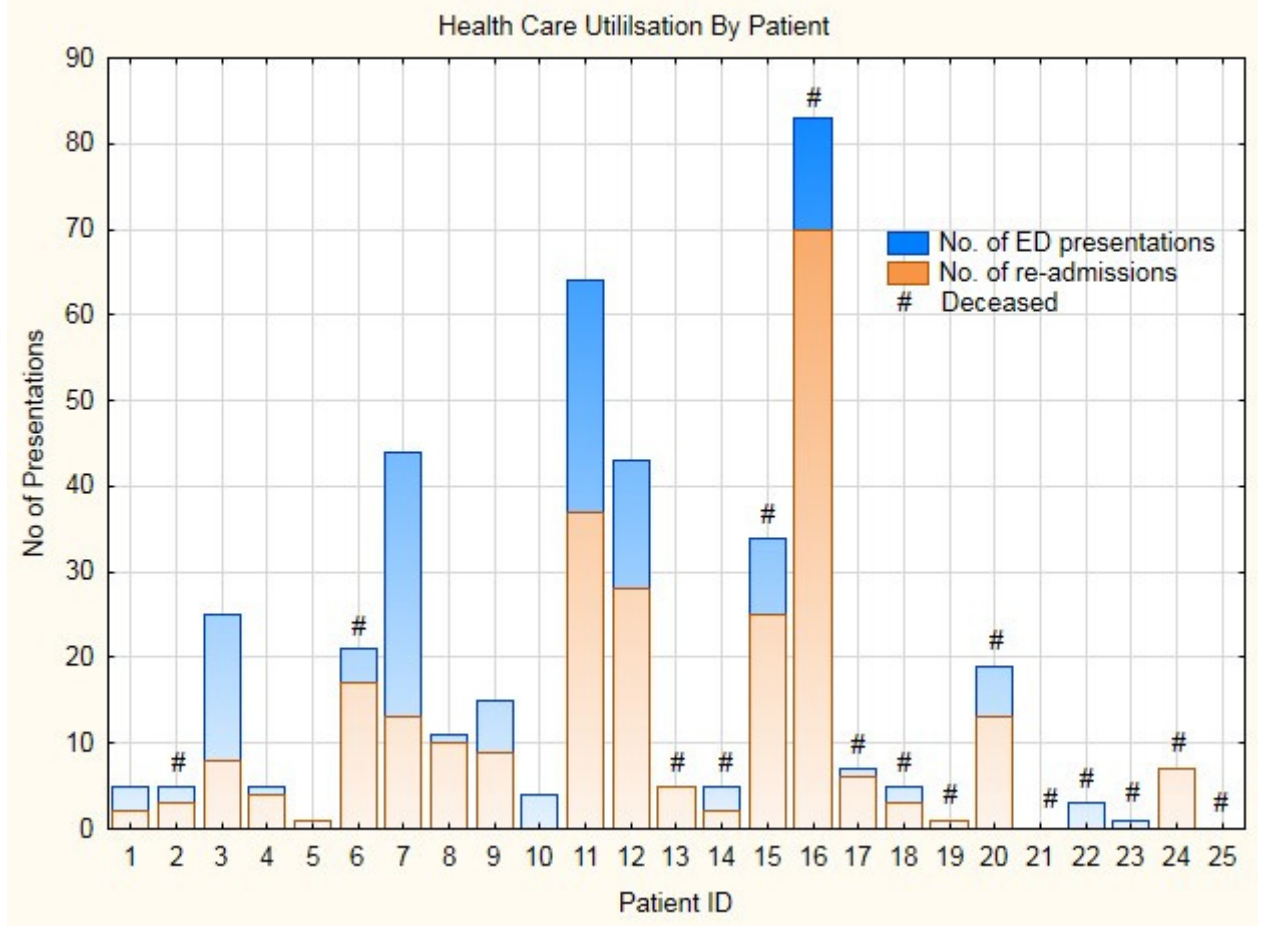

ED, emergency department.

Figure 4: Stacked plot health care presentations for eligible patients. 


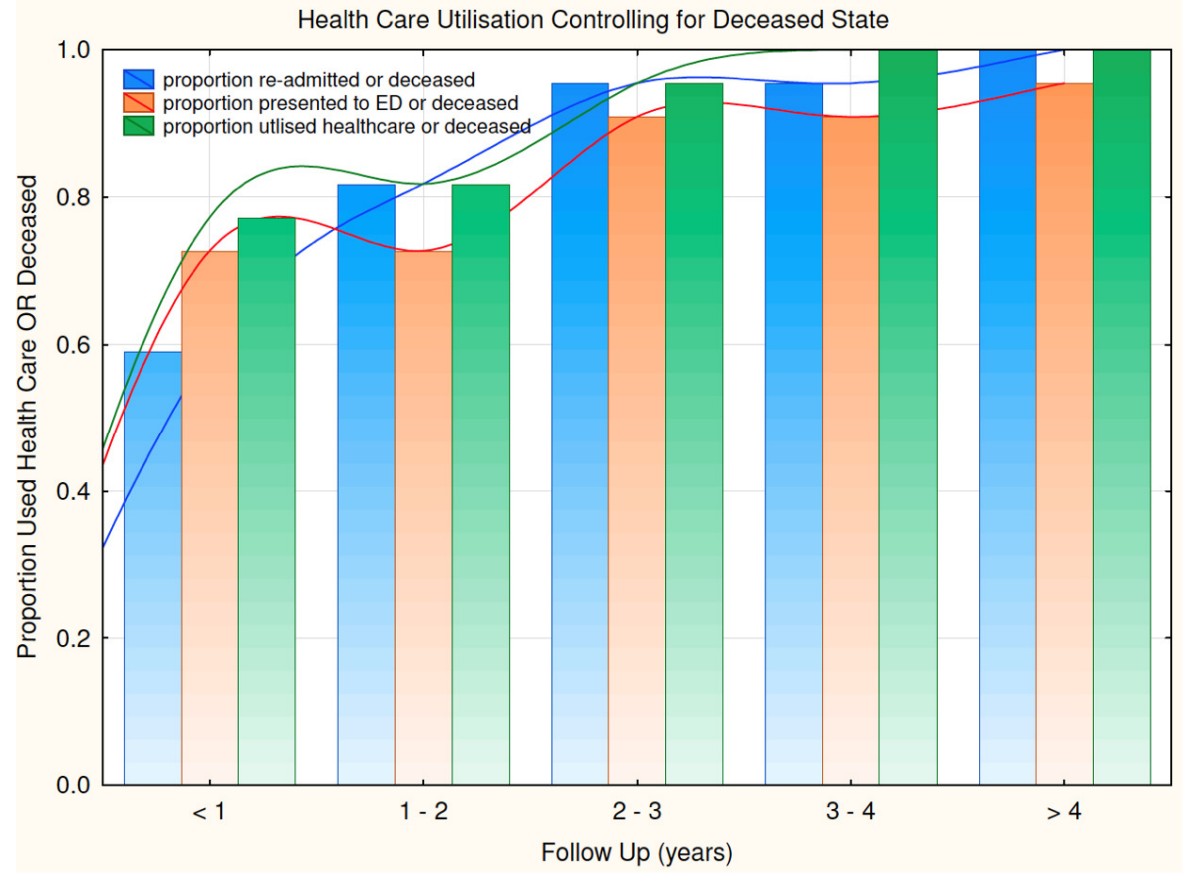

ED, emergency department.

Figure 5: Healthcare utilisation controlling for deceased status for eligible patients.

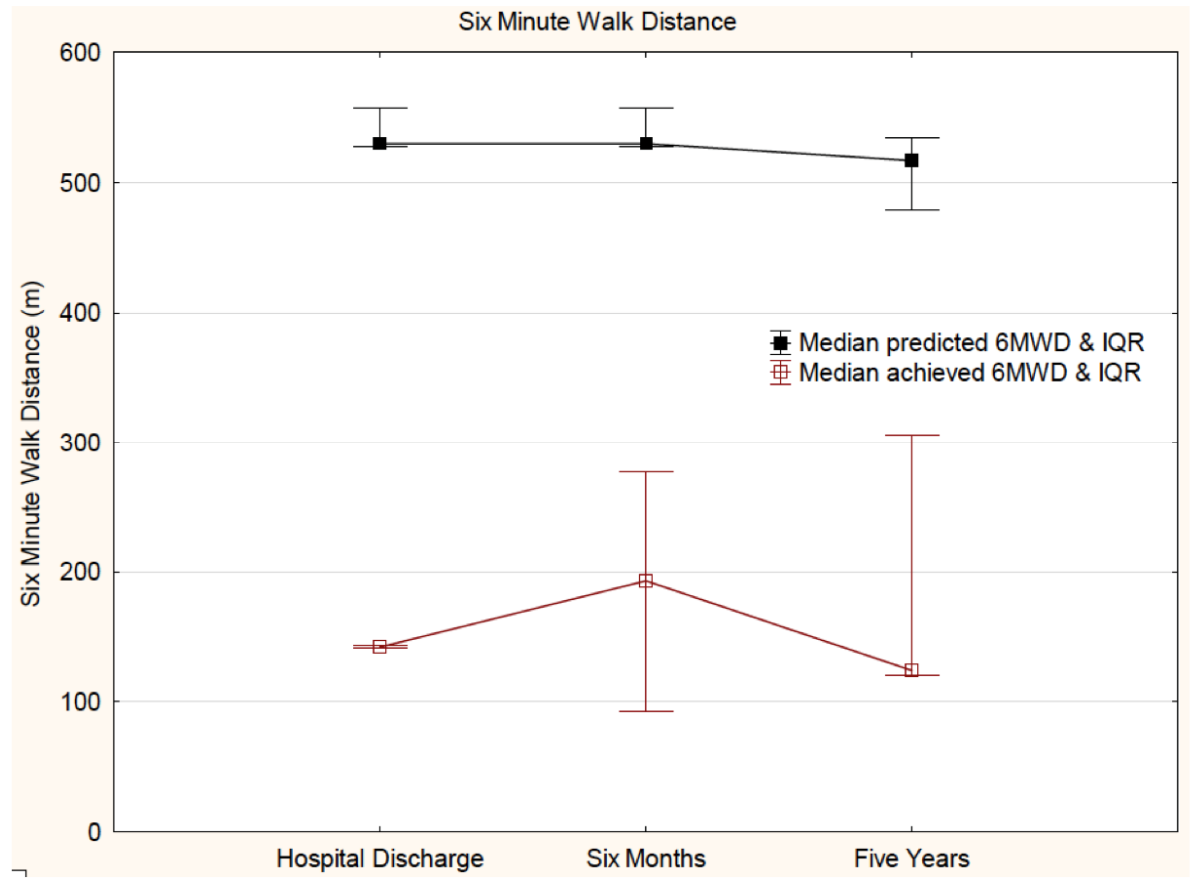

6MWD, 6-minute walk distance. IQR, interquartile range.

Figure 6: Mean predicted and achieved 6-minute walk distance for eligible patients. 
Therefore, despite a very low intra-ICU standardised mortality ratio (Table 1, Fig1), survivors of a critical illness are being resuscitated and rehabilitated only to succumb in the community with excess long-term morbidity and mortality in comparison to other follow-up studies. This could be viewed as a poor economic investment given the significant costs at the 'front end' of critical and acute care medicine, and suggests a need to increase investment at the primary health level. Expenditure per person on non-hospital secondary services for Indigenous Australians is about $57 \%$ of that for non-Indigenous people, suggesting that a large proportion of secondary and primary care occurs at acute care facilities, and this would be supported by the high rates of healthcare utilisation seen in this cohort ${ }^{4,27}$. This conclusion is supported by a recent article by Zhao and colleagues who modelled the economic benefits of eliminating Indigenous health inequality in the Northern Territory, reporting that closure of the gap would result in significant economic benefits ${ }^{26}$.

\section{Limitations}

This study has a number of limitations. The definition of 'patients at risk for future morbidity' was difficult to derive. There is little research around identifying patients at risk for future morbidity, and the only factors associated to date are age and duration of ICU admission ${ }^{8}$. Profound respiratory disease (acute respiratory distress syndrome) could be extrapolated as a risk factor from the work by Herridge and her group ${ }^{9,30}$. The authors' definition, therefore, used both a time component (more than 4 days), a level of physiological derangement (requiring ventilation), or apparent untreated disease processes (ICU admission with preceding hospital admission). This nevertheless limits the generalisability of these results.

Furthermore, the cohort inclusion criterion was surviving their initial critical illness. This was deliberately chosen since the ASH ICU standardised mortality ratio was low, and the anecdotal impression was that ICU mortality was falling, an impression subsequently borne out by the work of Kaukonen $^{31}$. Despite reasonably broad inclusion criteria, 2009 was a 'quiet' year, resulting in substantially fewer participants than originally envisaged. This is further compounded by the high post-hospital discharge mortality rate, which makes interpretation of the functional outcomes difficult. Compounding the difficulty in interpreting the 6minute walk distance is the lack of validation of this test in the Indigenous population.

Multivariate analysis would have been ideal in order to identify risk factors both for mortality and for the high rates of subsequent healthcare utilisation, but the numbers recruited were considered too small to undertake this analysis with any degree of confidence. This must be left to a subsequent larger study.

Measures of healthcare utilisation are difficult to interpret, particularly when there is a high mortality rate (since a deceased patient no longer uses health services) and there is no universally used tool ${ }^{32}$. The authors chose to use a measure (secondary healthcare presentation per year alive) that attempted to control for mortality, while also recognising that primary healthcare facilities are relatively under-used by Indigenous peoples ${ }^{27}$. Both ED presentations and re-admissions were included in this measure because Indigenous patients are hospitalised more frequently for preventable diseases, receive far more of their secondary medical care in hospitals and have less spent per person on Medicare services and the Pharmaceutical Benefits Scheme $e^{4,7,26,27}$. An interpretation of this data is that acute care facilities are providing more primary and preventative care services.

The present study's findings are limited by no measure of pre-index admission healthcare utilisation. Nor were GP, local clinic or planned outpatient presentations measured, which is likely to result in an underestimation of the healthcare utilisation rate reported in this study. However, between 2009 and 2014 there were between 38000 and 44500 presentations to the ASH ED, of whom approximately $30 \%$ required inpatient admission. Furthermore, over the same time period, the ASH averaged 42000 inpatient episodes per year ${ }^{33}$. Therefore, it would seem that this cohort of patients surviving a critical illness has a significantly higher health utilisation than the remaining local population. 
Finally, although this is a prospectively recruited longitudinal observational study, the unique features of this group make generalisability of these results to other critical care settings problematic.

\section{Conclusions}

There are very few prospectively recruited studies examining the outcomes of an ICU admission for a life-threatening illness. This study adds to the body of evidence that demonstrates that there are significant sequelae following a life-threatening illness, and should prompt further prospective studies of ICU survivors to better quantify these sequelae, in particular healthcare utilisation. As medical advances continue to improve, and there are more ICU survivors, clinicians, policy makers and administrators should be aware that the medium- to long-term sequelae of an ICU admission are significant and have major social and health economic consequences ${ }^{31}$.

Despite successfully supporting critically ill patients to ICU and hospital discharge there is a disproportionately high mortality rate in this regional cohort, particularly considering the relatively young age at admission. Given the large investment in healthcare resources during the index admission, and the ongoing high rates of healthcare utilisation, it is reasonable to conclude that there needs to be more emphasis on post-ICU interventions to reduce morbidity and mortality in this group.

\section{Acknowledgements}

This project was supported by a generous grant from the Norva Dahlia Foundation.

\section{References}

1. Secombe PJ, Stewart PC, Brown A. Functional outcomes in high risk ICU patients in Central Australia: a prospective case series. Rural Remote Health (Internet) 2013; 13(1): 2128. Available: www.rrh.org.au (Accessed 12 February 2013).
2. Australian Bureau of Statistics. Census QuickStats: Alice Springs Statistical Area Level 3. (Internet) 2011 Available: http://www. censusdata.abs.gov.au/census_services/getproduct/census/2011/ quickstat/70201 ?opendocument\&navpos $=220$ (Accessed 9 July 2015).

3. Australian and New Zealand Intensive Care Society (ANZICS) Centre for Outcomes and Resource Evaluation (CORE). ANZICS CORE Report. Melbourne: ANZICS, 2014.

4. Russell LM. Reports indicate that changes are needed to close the gap for Indigenous health. Medical Journal of Australia 2013; 199(11): 737-738

5. Commonwealth of Australia. Closing the Gap: Prime Minister's report 2015. (Internet) 2015 Available: http://www.dpmc. gov.au/sites/default/files/publications/Closing_the_Gap_2015_R eport_0.pdf (Accessed 8 July 2015).

6. Council of Australian Governments Reform Council. COAG Reform Council: Indigenous reform 2011-12: comparing performance across Australia. Report to the Council of Australian Governments. (Internet) 2013. Available: http://www.naccho.org.au/ download/aboriginal-health/COAG\%20reform\%20Counci 1\%20Indigemous\%20reform\%202011-2012.pdf (Accessed 8 July 2015).

7. Alford KA. Indigenous health expenditure deficits obscured in Closing the Gap reports. Medical Journal of Australia 2015; 203(10): 403.

8. Desai SV, Law TJ, Needham DM. Long-term complications of critical care. Critical Care Medicine 2011; 39(2): 371-379.

9. Herridge MS, Tansey CM, Matte A, Tomlinson G, DiazGranados N, Cooper A. Functional disability 5 years after acute respiratory distress syndrome. New England Journal of Medicine. 2011; 364(14): 1293-1304.

10. Liu V, Lei X, Prescott HC, Kipnis P, Iwashyna TJ, Escobar GJ. Hospital readmission and healthcare utilization following sepsis in community settings. Journal of Hospital Medicine 2014; 9(8): 502507.

11. Prescott HC, Langa KM, Liu V, Escobar GJ, Iwashyna TJ. Increased 1-year healthcare use in survivors of severe sepsis. American Journal of Respiratory and Critical Care Medicine 2014; 190(1): 62-69. 
12. Hill AD, Fowler RA, Pinto R, Herridge MS, Cuthbertson BH, Scales DC. Long-term outcomes and healthcare utilization following critical illness - a population-based study. Critical Care 2016; 20(1): 1-10.

13. Secombe PJ, Brown A, Kruger PS, Stewart PC. Lipid profiles and persisting inflammation following critical illness in a Central Australian population: a prospective longitudinal observational study. Internal Medicine Journal 2013; 43(4): 445-449.

14. ANZICS Centre for Outcome and Resource Evaluation. Intensive care resources and activity in Australia and New Zealand activity report 2013/14. Melbourne: ANZICS, 2014.

15. Enright PL. The six-minute walk test. Respiratory Care 2003; 48(8): 783-785.

16. Enright PL, Sherrill DL. Reference equations for the six-minute walk in healthy adults. American Journal of Respiratory and Critical Care Medicine. 1998; 158(5 Pt 1): 1384-1387.

17. Solway S, Brooks D, Lacasse Y, Thomas S. A qualitative systematic overview of the measurement properties of functional walk tests used in the cardiorespiratory domain. Chest 2001; 119(1): 256-270.

18. Department of Health and Aging. National Program Guidelines for the Home and Community Care Program. Canberra: Department of Health and Aging, 2007.

19. Charlson ME, Pompei P, Ales KL, MacKenzie CR. A new method of classifying prognostic comorbidity in longitudinal studies: development and validation. Journal of Chronic Disease 1987; 40(5): 373-783.

20. Needham DM, Scales DC, Laupacis A, Pronovost PJ. A systematic review of the Charlson comorbidity index using Canadian administrative databases: a perspective on risk adjustment in critical care research. Journal of Critical Care 2005; 20(1): 12-19.

21. Nesseler N, Defontaine A, Launey Y, Morcet J, Malledant Y, Seguin P. Long-term mortality and quality of life after septic shock: a follow-up observational study. Intensive Care Medicine 2013; 39(5): 881-888

22. Hicks PR, Mackle DM. Cause of death in intensive care patients within 2 years of discharge from hospital. Critical Care and Resuscitation 2010; 12(2): 78-82.
23. Hill K, Dennis DM, Patman SM. Relationships between mortality, morbidity, and physical function in adults who survived a period of prolonged mechanical ventilation. Journal of Critical Care 2013; 28(4): 427-432.

24. Williams TA, Dobb GJ, Finn JC, Knuiman MW, Geelhoed E, Lee $\mathrm{KY}$, et al. Determinants of long-term survival after intensive care. Critical Care Medicine 2008; 36(5): 1523-1530.

25. Australian Institute of Health and Welfare. Mortality inequalities in Australia: 2009-2011. Canberra: AIHW, 2014.

26. Zhao Y, Vemuri SR, Arya D. The economic benefits of eliminating Indigenous health inequality in the Northern Territory. Medical Journal of Australia 2016; 205(6): 266-269.

27. Wakerman J, Shannon C. Strengthening primary health care to improve Indigenous health outcomes. Medical Journal of Australia 2016; 204(10): 363-364.

28. Murray CJ, Atkinson C, Bhalla K, Birbeck G, Burstein R, Chou D, et al. The state of US health, 1990-2010: burden of diseases, injuries, and risk factors. JAMA 2013; 310(6): 591-608.

29. Goodwin AJ, Rice DA, Simpson KN, Ford DW. Frequency, cost, and risk factors of readmissions among severe sepsis survivors. Critical Care Medicine 2015; 43(4): 738-746.

30. Herridge MS, Cheung AM, Tansey CM, Matte-Martyn A, DiazGranados N, Al-Saidi F. One-year outcomes in survivors of the acute respiratory distress syndrome. New England Journal of Medicine 2003; 348(8): 683-693.

31. Kaukonen KM, Bailey M, Suzuki S, Pilcher D, Bellomo R. Mortality related to severe sepsis and septic shock among critically ill patients in Australia and New Zealand, 2000-2012. JAMA 2014; 311(13): 1308-1316.

32. Diehr P, Yanez D, Ash A, Hornbrook M, Lin DY. Methods for analyzing health care utilization and costs. Annual Review of Public Health 1999; 20: 125-144.

33. Alice Springs Hospital. 'ED presentations and admissions'. Alice Springs hospital admission data (unpublished). Alice Springs: Alice Springs Hospital, 2016. 